\title{
SELEÇÃO DE CONFIGURAÇÕES DE POÇOS DE PETRÓLEO BASEADA EM GERENCIAMENTO DE INTEGRIDADE, UTILIZANDO DIAGRAMA DE BLOCOS DE CONFIABILIDADE
}

\author{
Maurício Moraes Neves Jr \\ Universidade Federal Fluminense \\ Rua Passo da Pátria, 156, Bloco E, São Domingos, Niterói, RJ \\ mauriciomoraes@id.uff.br \\ Gilson Brito Alves Lima \\ Universidade Federal Fluminense \\ Rua Passo da Pátria, 156, Bloco E, São Domingos, Niterói, RJ \\ glima@id.uff.br \\ Danilo Colombo \\ Universidade Federal Fluminense \\ Rua Passo da Pátria, 156, Bloco E, São Domingos, Niterói, RJ \\ colombo.danilo@petrobras.com.br

\section{Danilo Taverna Martins Pereira de Abreu \\ Universidade de São Paulo} \\ Av. Prof. Mello Moraes, 2231, Cidade Universitária, São Paulo, SP \\ danilo.abreu@usp.br

\section{Juliedson Serigati Salvalagio} \\ Universidade Federal Fluminense \\ Rua Passo da Pátria, 156, Bloco E, São Domingos, Niterói, RJ \\ jsalvalagio@id.uff.br
}




\title{
RESUMO
}

Para a produção de um campo de petróleo, uma das tarefas mais importante é selecionar as configurações de poço que tragam melhores resultados financeiros e níveis de segurança adequados para o desenvolvimento do projeto. Porém, a economicidade e segurança devem ser observadas num horizonte que corresponda a todo seu ciclo de vida do poço.

O custo para construção de um poço de petróleo está diretamente relacionado ao tempo de construção, já que a maior parcela de seu custo está ligada aos valores das diárias da sonda de perfuração. A engenharia de poço tem feito grandes avanços nos últimos anos no desenvolvimento de novas tecnologias e novos projetos de poço que garantam a redução do tempo de construção dos poços e, portanto, do custo do mesmo. Porém, surge a dúvida se projetos mais arrojados são econômicos ao se levar em consideração toda a vida útil do poço, incluindo Capex e Opex.

O objetivo deste trabalho é analisar a confiabilidade e manutenabilidade de quatro configurações de poços ao longo da vida útil utilizando um Diagrama de Blocos de Confiabilidade (RBD- Reliability Block Diagram) e simulação de Monte Carlo. Esta simulação foi utilizada para avaliar a demanda de workovers (manutenção) para o poço ao longo de 27 anos de produção. Desta forma, espera-se unir os gastos de construção e manutenção dos poços para avaliar qual dos quatros projetos apresenta a melhor vantagem econômica.

Palavra-chave: Configuração de Poços; Integridade; Diagrama de Blocos de Confiabilidade (DBC)

\begin{abstract}
One of the most important tasks for the production of an oilfield is to select well configurations that bring the best financial results and safety levels appropriate for the project development. However, the economy and safety must be observed in a horizon that corresponds to the whole life cycle of the well.

The cost to build an oil well is directly related to the construction time, since most of its cost is linked to the daily values of the drilling rig. Well engineering has made great technological advancements in recent years in the development of new technologies and new well designs that will reduce the well construction time and thus the cost of the well. However, the question arises whether bolder designs are economical when considering the all life of the well, including Capex and Opex.

The objective of this paper is to analyze the reliability and maintainability of four well configurations over their lifetime using a Reliability Block Diagram (RBD) and Monte Carlo simulation. This simulation was used to assess the demand for workovers over 27 years of production. Thus, it is expected to combine the costs of construction and maintenance of wells to assess which of the four projects has the best economic advantage.
\end{abstract}

Keywords: Well design; Integrity; Reliability Block Diagram (RBD) 


\section{INTRODUÇÃ̃}

O petróleo vem sendo utilizado pelo homem como fonte de combustível desde o início da era cristã, sob diferentes denominações, e hoje, sua utilização é encontrada no dia a dia das sociedades atuais sendo a principal fonte de energia do mundo. $\mathrm{O}$ fornecimento mundial de energia proveniente de óleo e gás corresponde aproximadamente $60 \%$ de todas as fontes de energia (ALAWAD e MOHAMMAD, 2016).

De acordo com o anuário estatístico brasileiro de petróleo, o volume de petróleo produzido no mundo em 2017 foi de 92,6 milhões de barris por dia. Já o consumo mundial de petróleo em 2017 totalizou 98,2 milhões de barris/dia, após aumento de 1,8\% (1,7 milhão de barris/dia) em comparação a 2016, segundo fonte ANP (2018).

Este consumo crescente levou a uma gradativa busca de jazidas, avançando em cenários offshore cada vez mais restritos e complexos, trazendo consigo uma série de riscos, tanto no ponto de vista de segurança, como os vazamentos, como do ponto de vista econômico, como as perdas de produção, gastos com manutenção. Isto leva a um aumento de demanda por novos modelos que auxiliem no gerenciamento de tais riscos (COLOMBO, 2018).

Garantir a integridade, tanto durante as fases de construção quanto nas fases de produção dos poços de petróleo, tem um impacto relevante na imagem das companhias atuantes na indústria, o que por sua vez possui uma influência significativa em seu desempenho comercial (FONSECA, 2012).

A barreira de segurança é um item fundamental para manter a integridade do poço, pois é ela quem irá impedir o vazamento dos fluidos para o meio ambiente. No Brasil, a agência nacional de petróleo - ANP publicou em sua resolução $n^{\circ} 46 / 2016$, um regulamento que versa sobre integridade de poços - (SGIP - Sistema de Gerenciamento de Integridade de Poços), em que define um conjunto solidário de barreiras - CSB, como um conjunto de um ou mais elementos de barreira com o objetivo de impedir o fluxo não intencional de fluidos da formação para o meio externo e entre intervalos no poço, considerando todos os caminhos possíveis. Atualmente, a avaliação da integridade é feita de maneira determinística, pela contagem do número de CSBs independentes disponíveis no poço.

Uma das formas de evitar vazamentos durante a fase de produção, devido a falha de integridade, é realizar intervenções de manutenção (Workovers), que mantenham os poços em situações seguras e com barreiras de segurança sobressalentes. Poços submarinos de lâmina d'água profunda e com maiores classes de pressão, possuem agravantes que dificultam a resposta a vazamentos e a atenção para a segurança durante o projeto de poços deste tipo deve ser ainda mais relevante. Adicionalmente, o custo relativo à construção e manutenção destes poços em projetos de Desenvolvimento da Produção deve ser levado em conta.

A prevenção de vazamentos de petróleo para o meio ambiente é, portanto, uma importante característica de poços de petróleo, e é um parâmetro que deve ser levado em consideração em seu projeto.

Um avanço em relação a esse conceito qualitativo de conjunto solidário de barreiras (CSB), consiste em mudar a abordagem para termos probabilísticos, cujos modelos permitem não só a quantificação da probabilidade de falha dos sistemas de interesse, mas também a estimativa de parâmetros de desempenho, tais como os custos associados à manutenção e tempo de produção parada para reparos. Inclusive, tais informações podem ser implementadas para a tomada de decisão ainda em fase de projeto. Por exemplo, se há a adoção de configurações de poço cuja construção é mais rápida e barata em detrimento de 
opções que minimizem os custos operacionais ao longo do ciclo de vida, pode-se estar incorrendo em custos adicionais a longo prazo, mesmo que não intencionalmente.

Vários trabalhos (CORNELIUSSEN, 2006; FONSECA 2012; ZANETTI, 2014; BOUÇAS, 2017; HAALAND 2017, ZHEN, 2018), tem sido desenvolvido nos últimos anos para avaliação de integridade e risco de poços de petróleo durante a fase de produção (COLOMBO, 2018). No entanto, é encontrado uma lacuna na literatura sobre uma abordagem de análises de integridade de poço no nascedouro do projeto, durante $\mathrm{o}$ planejamento da configuração do poço a ser aplicado no desenvolvimento daquele campo.

Considerando que existem diferentes cenários de poços a serem analisados para o desenvolvimento de um campo de petróleo, faz-se necessário uma comparação das possíveis configurações de poços entre si. Essa comparação permite avaliar seus níveis de disponibilidade e, consequentemente, de segurança operacional proporcionando uma comparação dos poços independente de suas configurações.

Motivado pelo exposto acima, esse trabalho propõe a comparação entre quatro diferentes configurações de poços, quanto ao número de manutenções devido a intervenções de workovers, respeitando critérios de probabilidade de perda de integridade préestabelecidos.

Para a análise foi utilizada a técnica de Diagramas de Blocos de Confiabilidade combinada ao método de Monte Carlo para simulação. Essa combinação de técnicas tem como principal vantagem a versatilidade, uma vez que permite a incorporação em um único modelo de todos os aspectos desejados destacados, como a previsão da probabilidade de falha, reparos, inspeções e testes. No entanto, a análise suportada pelo método de Monte Carlo demanda um grande número de repetições sucessivas para atender aos requisitos de precisão dos cálculos, o que a torna custosa computacionalmente. Portanto, nos modelos desenvolvidos foram adotados RBDs somente com os elementos mais relevantes para a confiabilidade do poço, a fim de não o inviabilizar computacionalmente.

\section{CONFIGURAÇÕES DE POÇOS ANÁLISADAS}

Quatro configurações de poço foram estudadas nesse trabalho, a seguir:

A. poço convencional,

B. poço convencional considerando revestimento intermediário como barreira backup,

C. poço packoffless com BMA (Barreira Mecânica de Anular).

D. poço packoffless convencional e

A configuração A se refere a um poço convencional em termos construtivos, com os revestimentos condutor, de superfície, intermediário e de produção. Essa configuração conta com o packoff como elemento de vedação entre o anular e o meio ambiente. A configuração $\mathrm{B}$ é idêntica à configuração $\mathrm{A}$, mas possui o revestimento intermediário como elemento contribuinte à integridade do poço.

Diferentemente das configurações $\mathrm{A}$ e $\mathrm{B}$, as configurações $\mathrm{C}$ e $\mathrm{D}$ não adotam o packoff (poço packoffless). Ambas são providas de liner de produção e liner packer. Em especial, a configuração $\mathrm{C}$ também conta com uma barreira mecânica anular (BMA) como elemento redundante de integridade em relação ao liner packer, conforme pode ser visto na figura abaixo. 

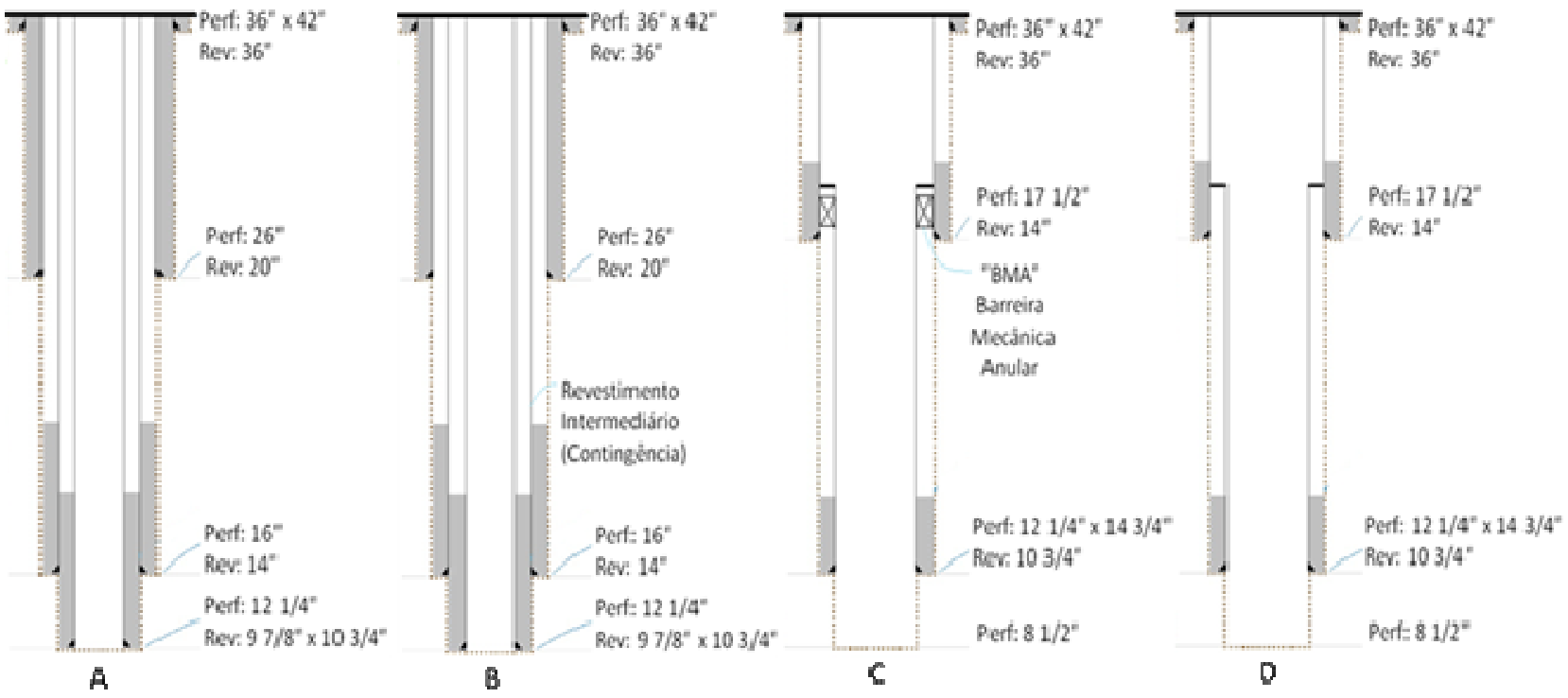

Figura 1 - Configurações estudadas de poços.

Portanto, nas configurações estudadas, as principais diferenças residem em aspectos relacionados à construção dos poços e não à completação desses. Dessa maneira, todos os poços possuem em comum os seguintes elementos relevantes à integridade

- Coluna de produção;

- $\quad$ Downhole safety valve (DHSV);

- $\quad$ Base adaptadora de produção (BAP) com duas válvulas de acesso ou anular (AI1 e AI2);

- Válvulas da árvore de natal molhada (ANM), com as suas válvulas:

o $\quad$ Mestra de produção (M1);

o $\quad$ Lateral ou wing de prod. (W1);

o $\quad$ Mestra do anular (M2);

o $\quad$ Lateral ou wing do anular (W2);

o Crossover (XO);

- Válvula de gas-lift;

- $\quad$ Packer de produção (referido simplesmente como packer);

- $\quad$ Anel VX;

- Selos superior e inferior do tubing hanger.

\section{METODOLOGIA DE ANÁLISE}

Nessa seção será apresentada a metodologia de análise em três partes distintas. Inicialmente, na seção 3.1, será apresentado o conceito de diagrama de blocos (RBD) e seus respectivos blocos para cada configuração de poço estudada. Na seção posterior, 3.2 serão discutidos conceitos de probabilidade, estatística e confiabilidade que subsidiam o algoritmo utilizado para análise. E por último, na seção 3.3, será apresentado o algoritmo propriamente dito passo a passo, permitindo a compreensão da dinâmica da rotina de simulação desenvolvida e variáveis necessárias para isso. 


\subsection{ANÁLISE DE DIAGRAMA DE BLOCOS (ABD) OU RELIABILITY BLOCK DIAGRAM (RBD)}

Uma vez conhecidos os elementos de barreira de cada configuração do poço, foram construídos os seus RBDs. Um RBD é um diagrama que dispõe os componentes em formato de blocos de acordo com as suas relações funcionais em termos de confiabilidade. Nesses diagramas, os blocos (componentes) podem ser interpretados como partes de um caminho que leva um sinal de um ponto de partida a um ponto de chegada. Cada combinação de falhas de componentes que inviabilize a chegada do sinal do ponto de partida ao ponto de chegada representa uma falha do sistema como um todo.

Introduzido o conceito de RBD, apresenta-se então os RBDs construídos para o estudo, da Figura 3.1 à Figura 3.4. Partindo-se da circunferência branca à esquerda do diagrama (ponto de partida do RBD), os blocos constituem o caminho até a circunferência preta à direita (ponto de chegada do RBD). A falha em percorrer um único caminho significa a perda parcial de integridade do poço, mas não necessariamente um vazamento, uma vez que inviabiliza apenas um dos CSB. A falha simultânea em todos os caminhos possíveis, por sua vez, leva à perda da capacidade de conter o vazamento de hidrocarbonetos.

O RBD da configuração A - vide figura 3.1 - possui os blocos de revestimento de produção cimentado, revestimento de produção livre e packoff constituindo um caminho único de partida. Como esses blocos estão posicionados em série no diagrama, uma falha em qualquer um desses blocos significa a perda do caminho inferior do RBD, o que é equivalente à comunicação do anular $\mathrm{A}$ do poço com o meio ambiente. A configuração $\mathrm{B}$, ilustrada na figura 3.2, por sua vez, possui em adição um caminho paralelo representado pelo revestimento intermediário, ou seja, uma redundância. Isso significa que se houver nessa configuração um vazamento pelo revestimento de produção ainda há o revestimento intermediário para conter a comunicação com o meio ambiente.

Diferentemente das configurações A e B, a configuração C, apresentada na Figura 3.3, possui como elementos de barreira no caminho inferior o liner de produção, o liner packer, mais a redundância de um BMA (Barreira Mecânica de Anular). Por fim, a configuração D - vide Figura 3.4 - assume a construção de um poço semelhante ao da configuração $\mathrm{C}$, mas sem a redundância do BMA e, portanto, sua falha simples pode levar à perda desse trecho do caminho do RBD.

\subsection{CONCEITOS DE PROBABILIDADE, ESTATÍSTICA E CONFIABILIDADE}

Uma vez construídos os RBDs, é possível extrair os caminhos críticos mínimos de falhas (cut sets mínimos) do sistema, ou seja, as combinações de falhas de componentes que, se ocorrerem simultaneamente, levam à falha do sistema como um todo. Essas falhas servem de input para as demais partes do procedimento de simulação.

Conhecidos os cut sets mínimos, a probabilidade de falha do sistema é dada por:

$$
P(f a l n \alpha)=\sum_{i=1}^{n} P\left(c_{i}\right)-\sum_{i=1}^{n n-1} \sum_{j=1}^{n} P\left(c_{i} \cap c_{j}\right)+\sum_{i=1}^{n-2} \sum_{j=i+1}^{n-1} \sum_{i=1}^{n} P\left(c_{i} \cap c_{i} \cap c_{k i}\right)+\cdots
$$

Onde $P\left(C_{i}\right)$ denota a probabilidade do $i$-ésimo cut set que, por sua vez, é dada por:

$$
P\left(C_{i}\right)=P\left(E_{1} \cap E_{2} \cap \ldots \cap E_{m}\right)=P\left(E_{1}\right) \cdot P\left(E_{2}\right) \cdot \ldots \cdot P\left(E_{m}\right)
$$

Em que $E_{1}, \ldots E_{m}$ são os $m$ eventos de falha de componentes que constituem o cut set mínimo em questão. 


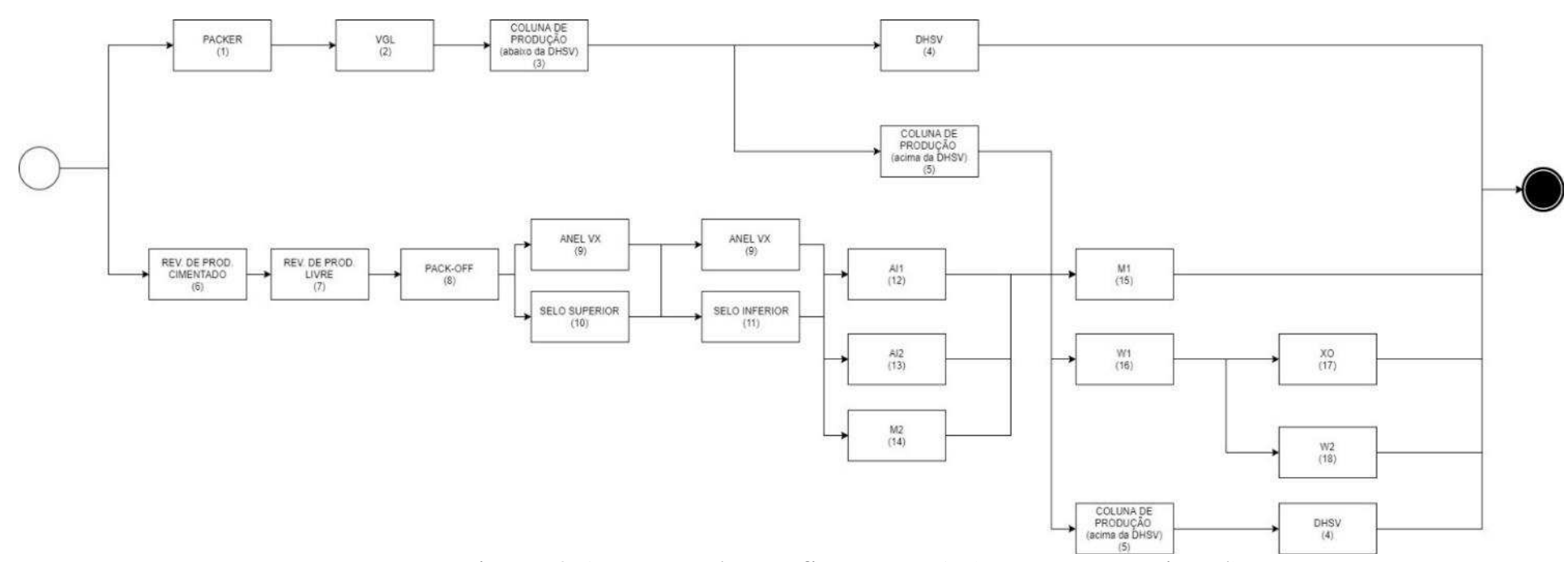

Figura 3.1 - RBD da configuração A (poço convencional)

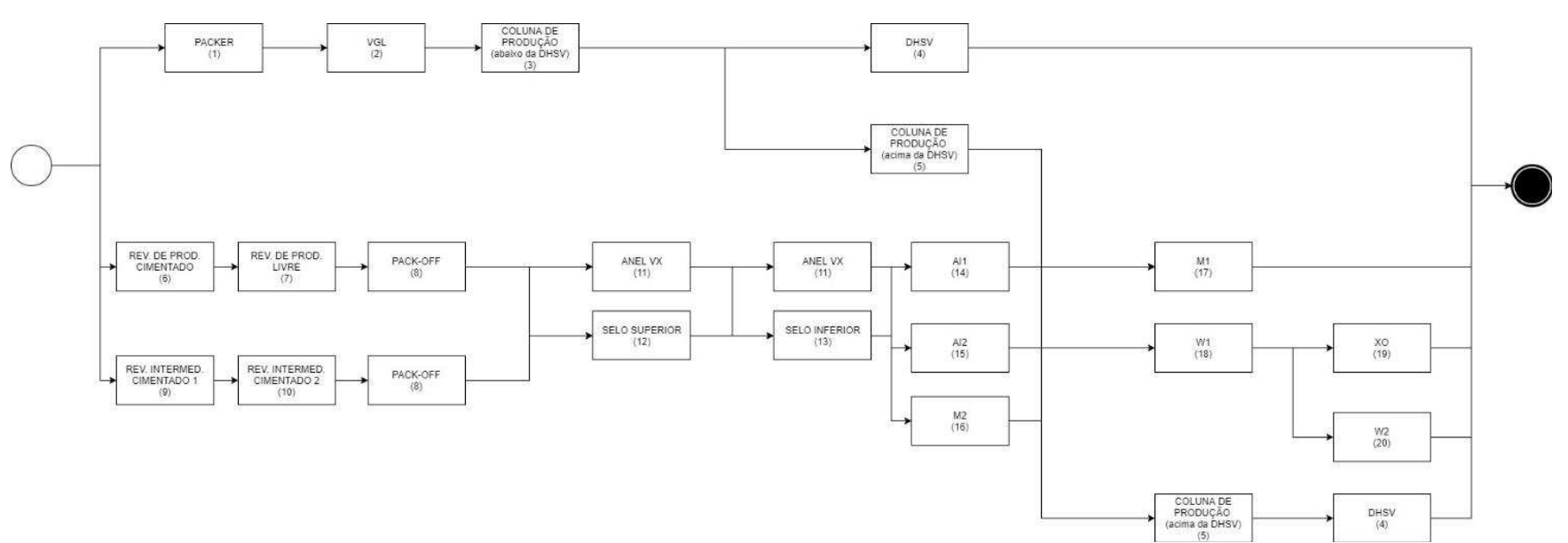

Figura 3.2 - RBD da configuração B (poço convencional com revestimento intermediário como barreira) 


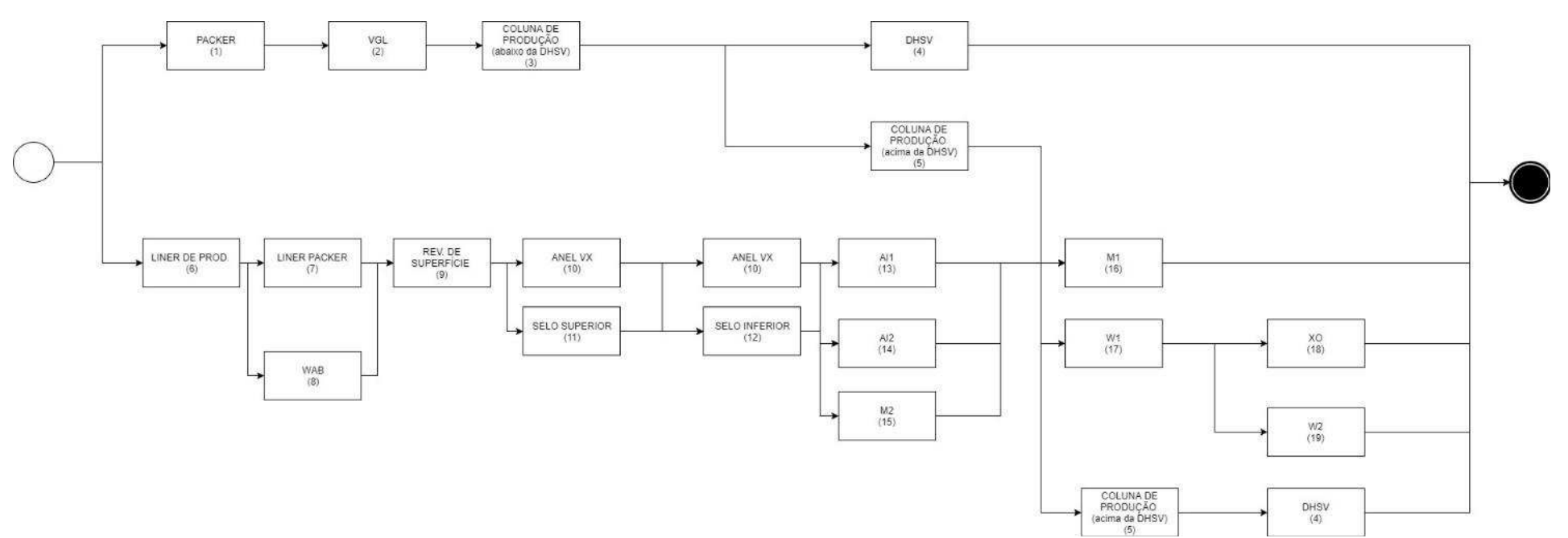

Figura 3.3. - RBD da configuração $\mathrm{C}$ (poço packofless com WAB)

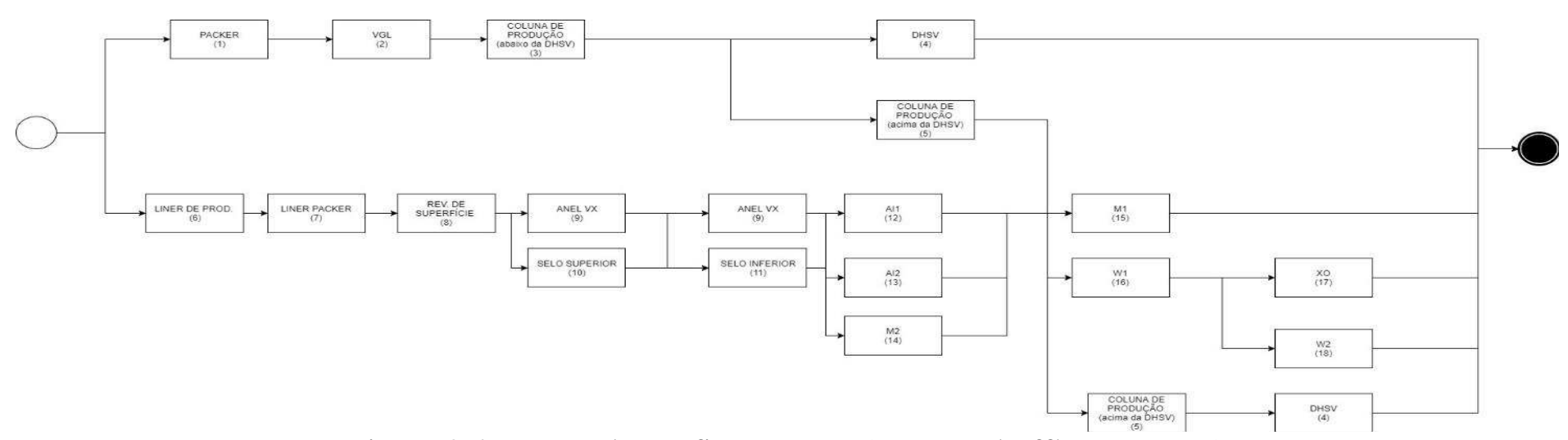

Figura 3.4 - RBD da configuração D (poço packoffless comum) 
As propriedades probabilísticas apresentadas acima são genéricas. Não se entrou no mérito até então de como calcular as probabilidades de falha dos componentes. Para modelos de falha dependentes do tempo, valem as seguintes relações:

Supondo que um componente possui uma função de densidade de probabilidade de falha, $f(t)$, regida por variável temporal (e, portanto, sempre positiva), a sua probabilidade de falha para um dado tempo de missão, $t$, denotada por $F(t)$, será dada pela probabilidade de que a variável aleatória que representa seu instante de falha, $T$, seja inferior ou igual a $t$. $F(t)$, recebe o nome de distribuição acumulada de falha. Matematicamente:

$$
F(t)=P(T \leq t)=\int_{0}^{t} f(\tau) d \tau, \quad t \in[0 ; \infty[
$$

Em específico, a distribuição de probabilidade de falha exponencial possui a sua função de densidade de probabilidade de falha e de distribuição acumulada dependentes de um único parâmetro, a taxa de falha, constante no tempo e denotada por $\lambda$. Essas funções são dadas respectivamente por:

$$
\begin{aligned}
& f(t)=\lambda \exp (-\lambda t), \quad t \in[0 ; \infty[ \\
& F(t)=1-\exp (-\lambda t), \quad t \in[0 ; \infty]
\end{aligned}
$$

Adicionalmente, uma vez conhecidas as distribuições de probabilidade de falha, também é de interesse estabelecer relações que permitam calcular o tempo estimado até a falha. Com essa motivação, são apresentadas mais duas colocações:

No caso de uma distribuição de probabilidade de falha exponencial, a inversa da função de distribuição acumulada é dada por:

$$
F^{-1}(p)=\frac{-\ln (1-p)}{\lambda}, \quad p \in[0 ; 1]
$$

Sorteando-se valores aleatórios de $p$, tais que $0 \leq p<1$, e utilizando a inversa da função de distribuição acumulada, gera-se uma amostra de tempos até a falha que tende a seguir a distribuição que os originou.

A distribuição exponencial foi adotada para todos os componentes nesse trabalho devido ao formato de dados utilizado. Essa é a única distribuição de probabilidade de tempo contínuo que possui a propriedade de ausência de memória. Matematicamente:

$$
P(X>s+t \mid X>s)=P(X>t), \quad s, t \geq 0
$$

Isso significa que a probabilidade condicional de a variável aleatória de interesse $(X)$ ser maior do que $s+t$, dado que $x$ é sabidamente maior que $s$ é a mesma probabilidade de $X$ ser maior que $t$ somente.

Em termos de confiabilidade, a propriedade de ausência de memória tem implicação direta nas premissas quanto ao comportamento de falha dos componentes. Segundo essa propriedade, por exemplo, se foi verificado que um componente não falhou até o instante $s$, então a sua probabilidade de falha projetada até o instante $s+t$ será equivalente a $F(t)$ e não a $F(s+t)$. Pragmaticamente falando, isso significa que o componente opera tão bom quanto novo após uma inspeção bem-sucedida. Apesar de essa premissa não ser realista para a maioria dos componentes, trata-se de uma das poucas abordagens viáveis quando só são conhecidas as taxas de falha dos componentes. Isso, entretanto, não impede modelos que adotam distribuições exponenciais de gerarem bons resultados, principalmente em análises comparativas. A sofisticação do modelo de falhas para representar a degradação de componentes exige o ajuste de outras distribuições de probabilidade de falha (e.g., Weibull), 
o que exige um banco de dados com tempos até falha detalhados, bem como o registro de não falhas.

\subsection{ALGORITMO: SIMULAÇÃO DA DINÂMICA DE FALHAS, REPAROS E INSPEÇÕES PARA CONFIGURAÇÕES DADAS DE POÇOS DE PETRÓLEO}

O algoritmo desenvolvido para a simulação foi dividido em duas partes, A e B, referentes respectivamente à sua inicialização (estabelecimento de parâmetros globais necessários) e simulação de Monte Carlo (onde os cálculos são efetivamente realizados). As rodadas de simulação de Monte Carlo descritas se aplicam a um único conjunto de valores exigidos na Parte A. Portanto, a avaliação do comportamento do sistema para diferentes configurações deve incluir as partes A e B da rotina de simulação e não apenas a parte B.

A descrição dos passos de cada parte está apresentada a seguir:

Parte A: inicialização do algoritmo.

1. Estabelecer a relação de cut sets mínimos da configuração, denotada por $\phi$.

2. Estabelecer o número de rodadas de simulação, $N$.

3. Seja $m$ o número de componentes (ou blocos) do RBD. Estabelecer as taxas de falha de cada componente, $\lambda_{i}, i=1, \ldots, m$.

4. Estabelecer o tempo de vida operacional do poço para análise, $t_{w 0}$.

5. Estabelecer a probabilidade admissível de blowout, $p_{\text {a adm }}$.

6. Estabelecer a lista com os tipos de reparos considerados e seus tempos ativos de reparo. Seja $r$ o número tipos de reparos, então $A R T_{i}, i=1, \ldots, r$, denotará o $i$-ésimo tempo ativo de reparo.

7. Estabelecer o tempo de programação e mobilização até o reparo, ${ }^{t_{m}}$.

Parte B: Simulação de Monte Carlo. O procedimento é repetido $N$ vezes:

1. Estabelecer o número esperado de inspeções $n_{\text {insp }}$. Inicialmente, $n_{\text {ims }}=0$.

2. Estabelecer o tempo de downtime total devido a reparos, $t_{\text {com }}$ time. Inicialmente, $t_{\text {downtime }}=0$.

3. Estabelecer o número esperado de cada tipo de reparo $n_{i}^{r * r}, i=1, \ldots, r$. Inicialmente, $n_{i}^{r p}=0, i=1, \ldots, r$.

4. Inicializar o tempo decorrido do sistema, $t=0$.

5. Enquanto $t \leq t_{w 0}$, repetir os passos de 6 a 11 . 
6. Estabelecer os tempos de falha, ${ }_{i}^{F}$ para cada um dos $m$ componentes $(i=1, \ldots m)$. Isso é feito sorteando ${ }^{m}$ números aleatórios entre 0 e 1 e aplicando a inversa da distribuição acumulada para cada componente segundo sua taxa de falha.

7. Projetar a curva de probabilidade de falha do conjunto de barreiras do poço de $t$ até $t_{w e}$, com base em $\phi$. Trata-se de uma coleção de pontos relacionando em ordem crescente a probabilidade de falha em diferentes instantes de tempo no intervalo $\left[t ; t_{w o}\right]$. A curva é construída com base em dois tipos de cálculo de probabilidade:

a. Para um componente recém-inspecionado, sem registro de falha, as probabilidades de falha utilizadas vão de um tempo de missão de 0 a $t_{v e}-t$, o que condiz com a ausência de memória da distribuição exponencial;

b. Para um componente não sujeito a inspeção, as probabilidades de falha utilizadas abrangem um tempo de missão de $t^{t} t_{v o}$.

8. Na curva de probabilidade, identificar o primeiro instante de tempo em que a probabilidade de vazamento é maior ou igual a $p_{\text {adm }}$, denotado por $t_{i n s p}$.

9. Verificar se ${ }^{t_{i}^{F}}>t_{\text {iwsy }}-t$. Em caso afirmativo, seguir para o passo 10. Em caso negativo, fazer $t=t+t_{\text {insp }}, n_{i n s p}=n_{\text {wnsp }}+1$ e voltar para o passo 5 .

10. Fazer $t=t+\varepsilon_{m o b}$ (contagem do tempo de mobilização até o reparo);

a. Seja $\bar{j}$ igual aos índices de reparos identificados como necessários no passo 9. Fazer:

b. $t=t+\Sigma_{j} A R T_{j}$

c. $t_{\text {downtime }}=t_{\text {downtime }}+\Sigma_{j} A R T_{j}$;

d. $n_{j}^{r e p}=n_{j}^{r e p}+1$.

e. Voltar para o passo5.

11. Armazenar para a rodada de simulação realizada os valores de $n_{\text {may }}, t_{\text {clows }}$ time $\mathrm{e}^{n_{j}^{m p}}$.

\section{RESULTADOS E DISCUSSÃO}

Para cada uma das quatro configurações de poço, a rotina de simulação apresentada na seção 3.3 foi realizada a partir dos seguintes parâmetros:

- Número de rodadas de Monte Carlo: 10 mil;

- Probabilidades admissíveis de blowout:

o $1,00 \mathrm{E}-05$;

o $3,00 \mathrm{E}-05$; 


$\begin{array}{ll}\text { o } & 5,00 \mathrm{E}-05 ; \\ \text { о } & 7,00 \mathrm{E}-05 ; \\ \text { о } & 9,00 \mathrm{E}-05 ; \\ \text { o } & 1,00 \mathrm{E}-04 ; \\ \text { o } & 3,00 \mathrm{E}-04 ; \\ \text { о } & 5,00 \mathrm{E}-04 ; \\ \text { о } & 1,00 \mathrm{E}-03 ;\end{array}$

- Tempo de vida operacional do poço: 30 anos;

- Escopos de manutenção segundo a Tabela 4.1;

- Taxas de falha dos componentes, fatores multiplicadores da probabilidade de blowout e características de inspeção segundo a tabela 4.2;

- Tempo de mobilização: 1 anos.

O fator multiplicador da probabilidade de blowout foi introduzido para diferenciar falhas que resultam em blowout daquelas que implicam em vazamentos menores e desprezíveis. Trata-se de um valor entre 0 e 1, que multiplica a probabilidade de falha do componente; conceitualmente representa a probabilidade condicional da ocorrência de um vazamento de proporções suficientemente grandes a ponto de resultar num blowout dada a ocorrência da falha do componente em questão. Esse parâmetro serve de input adicional ao modelo. Os fatores utilizados nesse trabalho foram propostos em relatório comercial, de circulação restrita.

As taxas de falha utilizadas, por sua vez, foram retiradas de bancos de dados comerciais.

Dadas as considerações acima, foram gerados resultados referentes ao número esperado de manutenções para atendimento das probabilidades máximas admissíveis de blowout; tempo de poço parado (downtime) devido a reparos; e número esperado de intervenções por cada tipo de intervenção apresentada na Tabela 4.1 ao longo dos 30 anos de vida operacional do poço.

A Figura 4.1 apresenta o número esperado de inspeções para cada configuração em função da probabilidade máxima admissível de blowout. As configurações A e C demonstram comportamento muito semelhante e suas curvas praticamente se sobrepõem. Para a manutenção de uma probabilidade máxima admissível de 1,0E-05, são previstas pouco mais de 90 inspeções. Já para a configuração D, cerca de 68 inspeções são necessárias para o mesmo nível de probabilidade admissível. Entretanto, à medida que a probabilidade admissível de blowout aumenta, as configurações $\mathrm{A}$ e $\mathrm{C}$ passam a demandar um número menor do que a configuração $\mathrm{D}$. A configuração $\mathrm{B}$, por sua vez, demanda um número significativamente menor e inspeções para se manter nos níveis mais baixos de probabilidade admissível, em torno de 10 inspeções previstas, mostrando-se ser a configuração de melhor desempenho nesse quesito. Em todas as configurações nota-se uma tendência à equalização do número esperado para as probabilidades admissíveis mais elevadas, mas que não atinge valores nulos (se mantêm num patamar de 3 inspeções).

Outro resultado, o número esperado de dias de downtime do poço para cada configuração em função da meta de probabilidade admissível de blowout é apresentado graficamente na Figura 4.2. De maneira abrangente, pode-se dizer que a configuração A tem o pior desempenho nesse aspecto, uma vez que demanda um grande número de dias de poço parado para reparo para diversas faixas de probabilidade admissível, sendo superada pela 
configuração D, entretanto, para a faixa de maiores probabilidades. A configuração B demonstra melhor desempenho para faixas intermediárias de valores de probabilidade admissível, mas é superada pela configuração $\mathrm{C}$ em ambas as extremidades.

Tabela 4.1- Escopos de manutenção do poço

\begin{tabular}{|c|c|c|}
\hline Tipo de manutenção & ART (dias) & Componentes reparados \\
\hline Substituição da válvula de ggs-lift & 14 & Válvula de ggs-lifts \\
\hline Substituição da árvore de natal molhada & 30 & Válvulas M1, W1, M2, W2 e XO \\
\hline Troca da base adaptadora de produção & 24 & $\begin{array}{c}\text { Packoff se los do tubing hanger, ane IVX e } \\
\text { válvulas Al1 e Al2 }\end{array}$ \\
\hline $\begin{array}{l}\text { Troca da coluna de produção (poço } \\
\text { convencional) }\end{array}$ & 120 & \multirow{2}{*}{$\begin{array}{c}\text { Racker de produção, coluna de produção, } \\
\text { DHSV }\end{array}$} \\
\hline $\begin{array}{l}\text { Troca da coluna de produção (poço } \\
\text { Rackofflesss) }\end{array}$ & 67 & \\
\hline
\end{tabular}

Tabela 4.2- Taxas de falha e fatores multiplicadores da probabilidade de blowout utilizados na simulação

\begin{tabular}{|c|c|c|c|}
\hline Componente & $\begin{array}{c}\text { axa de falha } \\
(1 / \mathrm{h})\end{array}$ & $\begin{array}{l}\text { Fator multiplicador da } \\
\text { probabilidade de blowout }\end{array}$ & $\begin{array}{c}\text { Falha detectável por } \\
\text { inspeção? }\end{array}$ \\
\hline AnelVX & $1,50 E-07^{(1)}$ & 0,1 & Sim \\
\hline $\begin{array}{c}\text { Coluna de produção (abaixo da } \\
\text { DHSV) }\end{array}$ & $9,00 \mathrm{E}-07^{(2)}$ & 0,5 & Sim \\
\hline $\begin{array}{c}\text { Coluna de produção (acima da } \\
\text { DHSV) }\end{array}$ & $1,50 \mathrm{E}-08^{(2)}$ & 0,5 & Sim \\
\hline DHSV & $5,07 \mathrm{E}-07^{(2)}$ & 0,1 & Sim \\
\hline Liner de produção & $8,20 \mathrm{E}-08^{(s)}$ & 0,1 & Não \\
\hline Liner packer & $7,30 \mathrm{E}-08^{(3)}$ & 0,5 & Não \\
\hline Packer & $7,30 \mathrm{E}-08^{(2)}$ & 0,5 & Sim \\
\hline Packoff & $6,00 E-08^{(4)}$ & 0,1 & Sim \\
\hline $\begin{array}{c}\text { Revestimento de produção } \\
\text { cimentado }\end{array}$ & $8,20 \mathrm{E}-08^{(2)}$ & 0,1 & Não \\
\hline Revestimento de produção livre & $8,20 \mathrm{E}-08^{(2)}$ & 0,1 & Não \\
\hline Revestimento intermediário & $8,20 \mathrm{E}-08^{(3)}$ & 0,1 & Não \\
\hline Selo inferior do tubing hanger & $1,50 E-07^{(1)}$ & 0,1 & Sim \\
\hline Selo superior do tubing hanger & $1,50 E-07^{(2)}$ & 0,1 & Sim \\
\hline Válvula Al1 & $3,70 \mathrm{E}-07^{(4)}$ & 0,1 & Sim \\
\hline VálvulaAl2 & $3,70 \mathrm{E}-07^{(4)}$ & 0,1 & Sim \\
\hline Válvula de gas-lift & $1,20 \mathrm{E}-05^{(z)}$ & 0,2 & Sim \\
\hline Válvula M1 & $1,37 \mathrm{E}-07^{(4)}$ & 0,1 & Sim \\
\hline Válvula M2 & $1,37 \mathrm{E}-07^{(4)}$ & 0,1 & Sim \\
\hline Válvula W1 & $1,37 \mathrm{E}-07^{(4)}$ & 0,1 & Sim \\
\hline Válvula W2 & $1,37 \mathrm{E}-07^{(4)}$ & 0,1 & Sim \\
\hline VálvulaXO & $1,37 \mathrm{E}-07^{(4)}$ & 0,1 & Sim \\
\hline WAB & $7,30 \mathrm{E}-08^{(\mathrm{s})}$ & 0,1 & Não \\
\hline
\end{tabular}

(a) Dado cetirado do estudg ES201236 - Risk analysis of Subsea Wells Completed with or without a intectace Controlled Subsurface Safety Valve (SCSSV) - Study Update and Revision 1

(2) Dado retirado do banco de dados Wellomaster

(s) Dado inferido a partir de componentes semelhantes

(4) Dado retirado do banco de dados Offshore Reliability Data(OREDA) 2015 


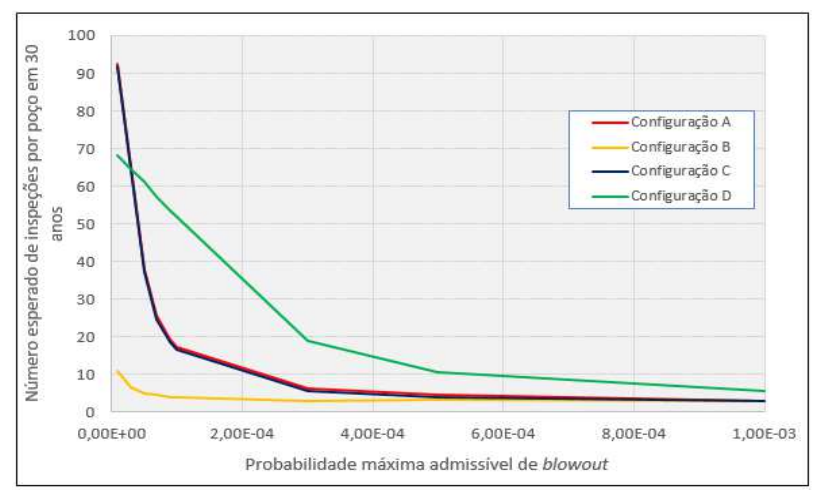

Figura 4.1 - Número esperado de inspeções

Nesse ponto, deve-se destacar um elemento comum ao resultado de tempo de downtime e os seguintes, referentes ao número previsto de reparos por categoria. Há alguns ruídos nos resultados para faixas de baixa probabilidade admissível de blowout. Em outras palavras, valores que deveriam ser estritamente decrescentes apresentam oscilação em determinadas faixas de valores. Isso se deve ao caráter numérico de avaliação do modelo apresentado. Tais ruídos são causados por eventos cuja baixa frequência de ocorrência demanda um número elevado de simulações para sua reprodução fidedigna. Portanto, a partir desse ponto, análises de tendências globais são mais representativas do que avaliações de peculiaridades locais dos gráficos.

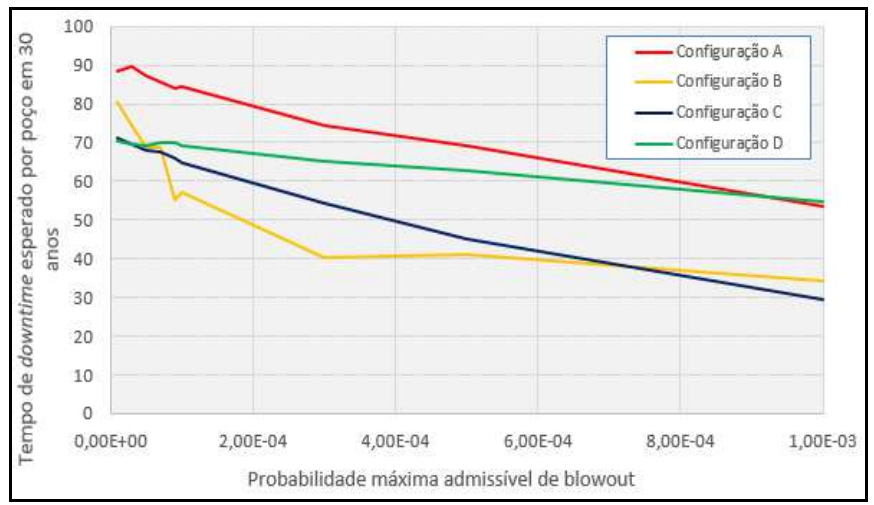

Figura 4.2 - Dias de downtime do poço para reparo devido ao tempo ativo de reparo

As taxas de falha foram multiplicadas por um fator variando de 0,001 a 10 . Para pequenos valores desse fator, pequenos ruídos numéricos foram notados, seguindo o problema de resolução numérica comentado anteriormente.

A análise para o packoff na configuração A está apresentada na Figura 4.3. O tempo de downtime esperado varia numa amplitude de menos de 10 dias, entre 75 e 84 dias. A probabilidade média de blowout, por sua vez, varia de 7,89E-05 a 3,91E-04. Já para a configuração B, os resultados estão apresentados na Figura 4.4. É notável como nessa configuração, diferentemente da anterior, o tempo downtime esperado é mais sensível, variando de 35 a 75 dias. A probabilidade de vazamento também sofre grande impacto: varia de 4,11E-06 a 3,21E-04, ou seja, flutua numa faixa de duas ordens de grandeza.

Quanto ao liner packer, os resultados para a configuração $C$ estão apresentados na Figura 4.5. Para essa configuração, variações na taxa de falha provocam variações 
desprezíveis tanto no downtime quanto probabilidade média de blowout. O tempo downtime esperado oscila entre 58 e 60 dias, enquanto a probabilidade média de blowout não avança além da faixa de 7,80E-05 e 8,00E-05. Diferentemente, a configuração D, cujos resultados estão apresentados na Figura 4.6, se mostra mais sensível a variações na taxa de falha do liner packer. A probabilidade média de blowout varia de 7,91E-05 a 7,79E-04 (avança em uma ordem de grandeza) e o downtime esperado varia de 59 dias para 69 dias assintoticamente. Tal diferença pode ser atribuída à redundância do liner packer na configuração C estabelecida pela BMA.

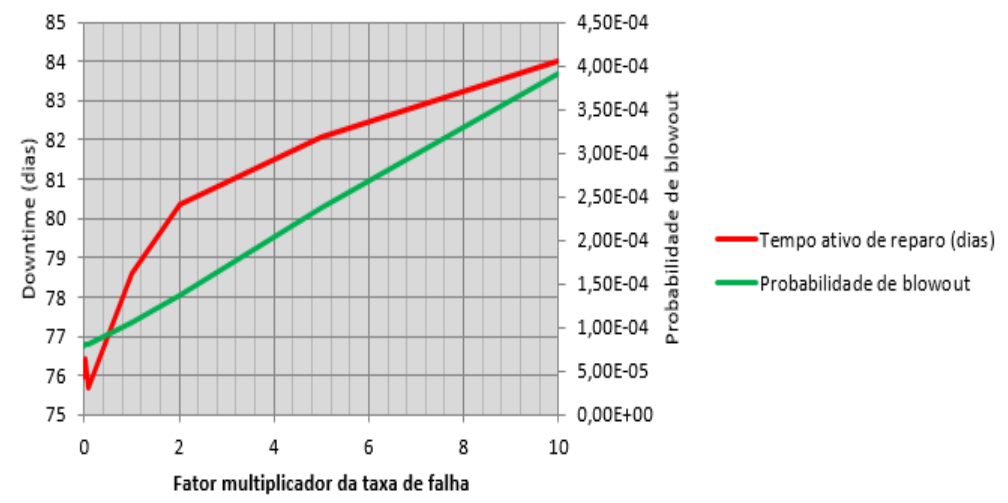

Figura 4.3 - Análise de sensibilidade do packoff na configuração A

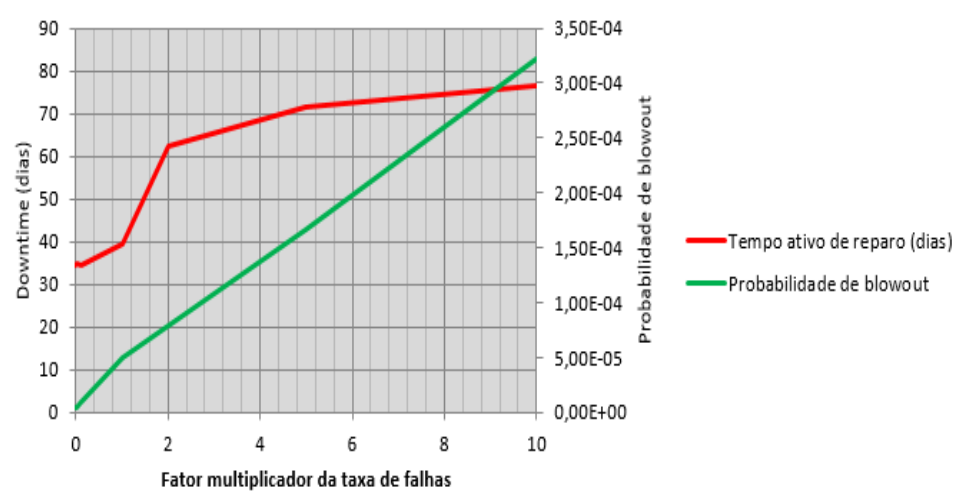

Figura 4.4 - Análise de sensibilidade do packoff na configuração B

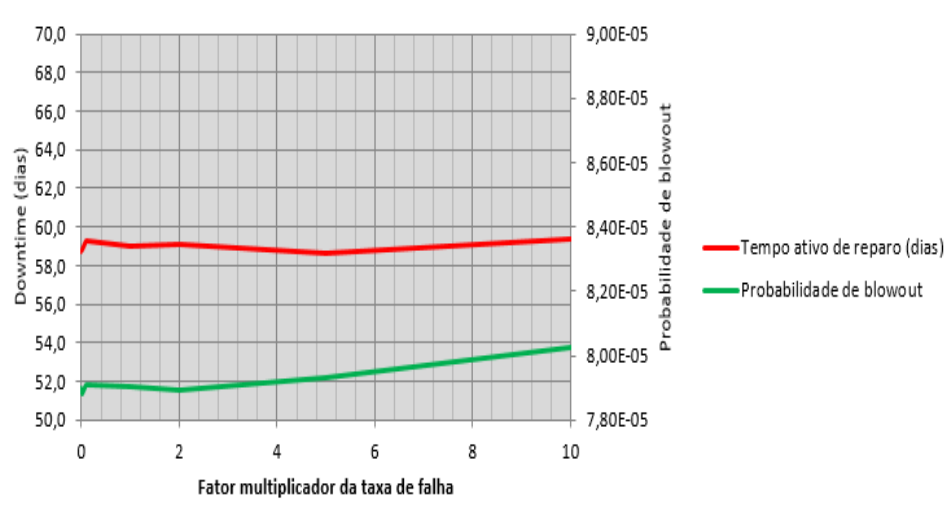

Figura 4.5 - Análise de sensibilidade do liner packer na configuração C (BMA contigencial) 


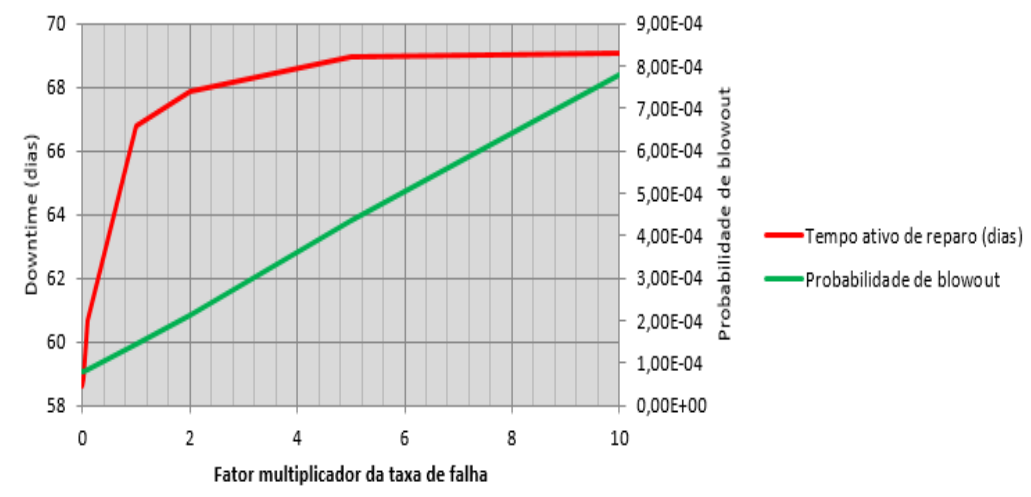

Figura 4.6 - Análise de sensibilidade do liner packer na configuração D

\section{CONCLUSÕES}

O trabalho descrito permitiu a comparação de quatro diferentes configurações de poços segundo as suas rotinas de reparos esperadas durante o ciclo de vida. Os fatores avaliados foram:

a) downtime esperado do poço devido às operações de reparo, expressado em dias;

b) número de inspeções necessárias;

Todos os resultados foram gerados em função de probabilidades máximas admissíveis de blowout.

Os escopos de manutenção considerados foram a substituição de coluna de produção, troca de válvula de gas-lift, substituição da BAP e substituição da árvore de natal.

De maneira geral, a configuração B (poço convencional com revestimento intermediário estanque), que leva em consideração o revestimento intermediário como barreira, apresentou o melhor desempenho na grande maioria dos aspectos. Esse desempenho se traduz num pequeno número de manutenções e inspeções necessário para garantir os requisitos de probabilidade máxima aceita para blowouts. Entretanto, essa aparente vantagem da configuração B é ofuscada pelo fato de uma das principais manutenções, que é a troca da coluna de produção, demandar aproximadamente o dobro de tempo nessa configuração em relação às configurações packoffless (configurações C e D) 120 dias versus 67 dias. Isso se traduz no desempenho semelhante das configurações $\mathrm{B}$ e $\mathrm{C}$ quanto ao tempo esperado de downtime do poço devido a reparos. Em especial, essa última configuração se beneficia pela redundância entre liner packer e BMA como elementos de barreira.

Estudos futuros podem aprimorar os resultados apresentados em alguns aspectos. Primeiramente, ainda há muitas incertezas quanto à taxa de falhas de elementos menos convencionais como a BMA. Melhorias na coleta de dados de falha, opiniões de especialistas e testes do fabricante contribuiriam para melhorar a fidedignidade dos valores apresentados. Além disso, o próprio modelo de diagrama de blocos pode ser aprimorado a fim de melhorar a eficiência de cálculo. Isso viabilizaria simulações de Monte Carlo com um número maior de rodadas e, portanto, mais precisas. Por fim, a inclusão de condições operacionais no modelo e seu impacto nas taxas de falha auxiliaria no aumento do grau de sofisticação. 


\section{REFERÊNCIAS BIBLIOGRÁFICAS}

[1] Abimbola, M.; Khan, F., (2016), "Development of an integrated tool for risk analysis of drilling operations", Process Safety and Environmental Protection, 102, pp. 421430.

[2] Agência Nacional de Petróleo, Gas Natural e Biocombustíveis (ANP). Resolução n46/2016: Regime de Segurança Operacional para Integridade de Poços de Petróleoe Gás Natural.

[3] Alawad, M, N. J.; Mohammad, H.A. "Engineering Management and Inspection Schedule of Petroleum Well Integrity". Journal of Emerging Trends in Engineering and applied Sciences (JETAS) 7 (3):109—117, 2016

[4] Colombo, D. "Proposição de um modelo Markoviano de apoio ao gerenciamento de riscos à integridade de poços submarinos". Dissertação de Mestrado - Universidade Federal Fluminense, Rio de Janeiro, 2018

[5] Colombo, D. Banco de Dados de Confiabilidade e Monitoramento em Tempo Real Aplicados à Integridade de Poços de Petróleo. Simpósio internacional de Confiabiliade (SIC), São Paulo, 2018.

[6] Colombo, D.; Lima, G.B.A.; Garcia, P.A.A.; Gavião, L.O.; Melo, P.F.F.F., (2017), "Modelagem Markoviana para a Análise de Confiabilidade de um Poço de Petróleo", Congresso Abrisco 2017, Rio de Janeiro, Brasil, 27-29 de novembro de 2017.

[7] Corneliussen, K. "Well Safety: Risk Controlin the Operational Phase of Offshore Wells. Tese Doutorado - The Norwegian University of Science and Technology, Trondhein, 2006

[8] Fonseca, T.C.; "Metodologia de Análise de Integridade para Projetos de Poços de Desenvolvimento da Produção", Dissertação de Mestrado - Universidade Estadual de Campinas, 2012.

[9] Fonseca, T.C.; Miura, K.; Mendes, J.R.P., (2013), "Well Integrity Analysis Applied to Workover Prediction", Offshore Technology Conference, Rio de Janeiro, Brazil, 29-31 October 2013.

[10] Halland, B; "Life Cycle Well Integrity and Realiability", Thesis (Master), University of Stavanger, 2017

[11] NORSOK, (2013), NORSOK Standard D-010: Well integrity in drilling and well operations, Rev. 4, June 2013, Standards Norway, Norway.

[12] SINTEF, (2015), Offshore Reliability Data (OREDA), 6th ed., Vol. 2 Subsea Equipment.

[13] WELLMASTER, Reliability of Well Completion Equipment - Phase VI Main Report. Trondhein, 2009.

[14] Zanetti, A.A.; “Avaliação Comparativa de Disponibilidade de Poços Submarino em diferentes cenários na fase Operacional”, Dissertação de Mestrado Universidade Federal do Rio de Janeiro, Rio de Janeiro, 2014. 